\title{
Perceptual Collapse: The Fusion of Spatially Distinct Tactile Cues Into a Single Percept
}

\author{
Steven G. Manuel ${ }^{+}$, J. Edward Colgate ${ }^{+}$, Michael A. Peshkin ${ }^{+}$, Roberta L. Klatzky* \\ ${ }^{+}$Northwestern University \\ Mechanical Engineering Department \\ 2145 Sheridan Road \\ Evanston, IL 60208 \\ *Carnegie Mellon University \\ Department of Psychology \\ Pittsburgh, PA 15213
}

\begin{abstract}
This work investigates how haptic percepts are combined across two fingertips. Two single-degree-of-freedom haptic interfaces were used to present virtual bumps to the thumb and index finger of subjects' right hand. As subjects slid the two interfaces from left to right while maintaining a fixed finger separation, they would encounter one bump with the index finger and one with the thumb. The objective bump locations were varied randomly, from spatially coincident to separated by slightly more than the fingertips. Subjects were asked to report the number of bumps in the objective world and the number of times they encountered each bump, and also to point to the locations of the bumps. We found that subjects exhibited a strong bias toward reporting a single objective bump in the virtual world. However, the percept varied from one bump encountered twice, when the two virtual bumps were nearly spatially coincident, to one bump encountered once, which occurred when the two virtual bumps were close to finger separation and therefore encountered nearly simultaneously. The latter result is evidence for a kinesthetic grouping phenomenon: temporally synchronized sensations at multiple fingers can be perceptually collapsed into a single percept in both time and space.
\end{abstract}

KEYWORdS: Perception and psychophysics; Neuroscience; Human performance.

INDEX TeRms: H.5.2 User Interfaces, H.5.2.i Interaction styles, H.5.2.o Theory and methods.

\section{INTRODUCTION}

It is well known that kinesthetic cues are sufficient for individuals to perceive illusory protrusions ("virtual bumps") on an otherwise flat surface [1]. In this paper we examine the situation in which two fingers on the same hand encounter virtual bumps and we show that, depending on bump separation and finger separation, either one or two bumps may be perceived. Thus, we provide evidence for grouping of percepts in a kinesthetic task. To the best of our knowledge, this is the first explicit demonstration of a Gestalt-like grouping principle in the kinesthetic domain.

Our interest in this problem stems from recent advances in surface haptic devices capable of providing force feedback to individual fingertips $[2,3]$. It is not a great stretch to imagine touch screens capable of applying forces (direction and magnitude) independently to each of multiple fingertips, and to ask the question: what new experiences will be enabled beyond

steven.manuel@u.northwestern.edu

colgate@northwestern.edu

peshkin@northwestern.edu

klatzky@cmu.edu

IEEE World Haptics Conference 2013

14-18 April, Daejeon, Korea

978-1-4799-0088-6/13/\$31.00 @2013 IEEE those available to single fingertips? We have already shown that two points of contact on opposing surfaces can actually mitigate a single-finger illusory percept [4], but our experience also suggests that when the forces on individual fingers are properly designed and coordinated, novel percepts may emerge. The most basic form of emergence may well be grouping, in which case, rather than experiencing objectively distinct percepts at each fingertip, the subject experiences only one objective percept. Grouping is a phenomenon that is well-known in Gestalt psychology and that has been extensively studied, especially in the context of vision. The next section provides a brief review of grouping principles.

\subsection{Grouping principles}

In vision, grouping is a process associated with intermediatelevel computations, where early sensory inputs are identified as regions, surfaces, and volumes. This process was extensively studied by the so-called "Gestalt" psychologists, who proposed a set of basic principles to describe how spatially distributed and often discrete visual elements are grouped together into perceptual units [5]. Subsequent work has expanded on these basic principles and clarified that they can occur at multiple levels of visual processing [6]. The relationships between multiple visual features that tend to support grouping include similarity, proximity, good continuation (common tangents), symmetry, closure (features that collectively enclose a region), and common fate (common motion) [5]. For example, a school of fish consists of several individual fish moving in unison, but what is perceived is a group of fish with a single common motion. An analogy can be made between elements of visual grouping, which often project onto non-contiguous regions of the retina [6], and haptically grouped physical features of objects that are detected by noncontiguous regions of skin, e.g., adjacent fingertips.

Haptic research that either explicitly studies Gestalt-like grouping principles or can be interpreted as such tends to involve the tactile domain, as opposed to the kinesthetic (or force feedback) domain, which is the focus of the current work (see Gallace et al. [7] for review). The grouping principles most relevant to the current work are grouping by similarity and common fate, so those will receive specific attention here. Chang [8], for instance, demonstrated parallels between the tendency to group by similarity vs. proximity in both haptic displays (consisting of patches of variable roughness) and equivalent visual displays. Overvliet [9] showed that haptic search times are shorter when line segments of similar orientation are scanned. Pawluk [10] indicated that movability of a 3-D object increases the tendency to interpret it as an object (figure) rather than a supporting surface (ground). It is possible that this result was affected by grouping by common fate, because multiple points of contact on the skin experience parts of a surface that move in unison. However the result falls short of demonstrating that multiple points of contact provide any additional benefit in terms of figure - ground discrimination or result in grouping of surfaces into a single object.

Two well-known haptic effects, saltation and funnelling, involve perceptual referral of spatially distinct tactile stimuli to a 
single location either on the skin or in external space between the stimulated locations $[11,12]$. However these effects have never been demonstrated in the kinaesthetic domain. Additionally, while funnelling may result in a stationary illusory percept, saltation inherently involves motion across the skin.

\section{Aims AND Hypotheses}

The present research attempts to demonstrate grouping of kinesthetic inputs, in the form of virtual bumps encountered by two fingers. Borrowing from the visual domain, which identifies grouping as an intermediate process that is used to grow unified regions from discrete elements, we propose that kinesthetic grouping will arise under conditions that promote the association of a single object with multiple points of stimulation. Constraints that should promote grouping, by suggesting a single emergent bump, are similarity of bump forces and spatio-temporal relations that, within kinesthetic noise, suggest a single source location in extrinsic spatial coordinates. If, in fact, two bumps are grouped into one, we assume that its perceived location will be assigned to a point between the fingers; in contrast, reporting the location associated with just one bump would suggest that it perceptually dominated the other. To address these hypotheses we conducted two experiments. Experiment 1 assessed participants' ability to localize single bumps presented to the index finger or thumb, while holding their fingers in an open pinch position. The measured error was intended to establish a lower bound on the expected error, which could be compared to the error induced by grouping multiple bumps. Experiment 1 was also intended to confirm that as bump spacing increases, subjects are able to determine that the bumps are rendered in separate locations. Experiment 2 directly assessed the tendency to group two bumps into one, by the criterion above, using the same finger posture.

\section{MATERIALS AND METHODS}

\subsection{Apparatus:}

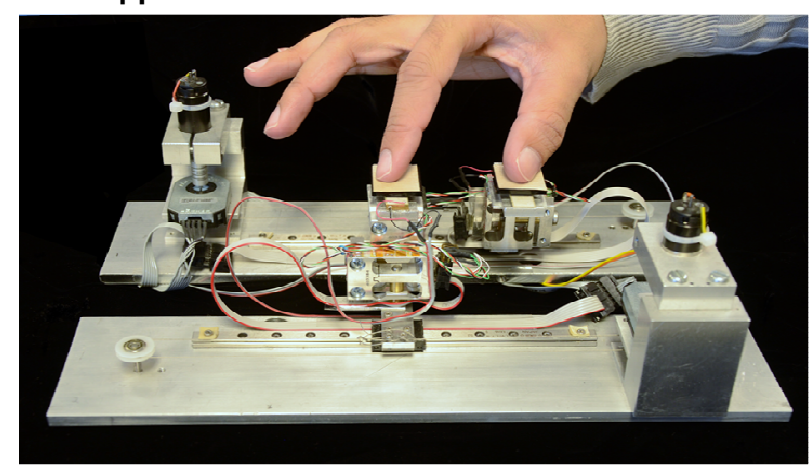

Figure 1. Haptic slider interface.

Our experimental setup consisted of two slider surfaces for two fingertips, constrained to slide along the same axis independently of one another. The total workspace reachable by both sliders together was $160 \mathrm{~mm}$ in length. Each slider surface was mounted on its own cable-driven linear bearing equipped with load cells to measure forces exerted by the finger on the slider surface on an axis normal to the surface as well as on the axis of travel.

Each slider was driven using a force control loop closed around the slider's lateral direction load cell, in order to mask the inertia and friction. The control loop operated at $1 \mathrm{kHz}$ and was computed on a PC/104 stack running an xPC Target real-time operating system. Automation of the experimental protocol was done on a PC running a Matlab script, communicating with the PC/104 stack. Visual feedback and user input was accomplished using a touch screen monitor located next to the slider apparatus.

Maxon RE-16 motors drove the sliders with up to $1 \mathrm{~N}$ of force, although the experiment required less than $0.7 \mathrm{~N}$ to render bumps effectively. We used Futek LSM250 parallelogram load cells with a full-scale reading of $1.1 \mathrm{~N}$. 320 grit sandpaper was used as the slider surfaces to ensure zero slip even at low levels of applied normal force.

Lateral forces (representing bumps) were rendered irrespective of the subject's applied normal force; the lateral force applied by the device was a function of slider position alone. We assumed a constant normal force for the purposes of computing lateral force given a desired bump size. This contrasts with an idealized frictionless bump in which lateral force would be proportional to the participant's applied normal force. Because the experiment requires subjects to keep their dominant arm elevated for extended periods, we used a forearm sling to reduce fatigue. The sling's support wires extended 8 feet upward to the ceiling, so the direction of tension during movement was largely insensitive to the position of the arm. In addition, to prevent visual cues from affecting responses, the subject's view of the device was obstructed by a metal sheet.

\subsection{Participants}

The participants included 2 males and 8 females between 18 and 59 years of age, who gave their informed consent. All were right handed, naïve to the purpose of the experiment and used their dominant hand for the experiment. All took part in both experiments.

\subsection{Protocol}

Subjects were told that the device exerts only lateral forces on each fingertip and that they would perceive small bumps along the path of the sliders despite the fact that the slider surfaces would not move in the vertical direction. Subjects were then allowed to feel a physical example of a bump: a plastic block with its upper surface formed into a one-dimensional Gaussian bump. Subjects placed their right hand in the sling and were instructed to explore a flat virtual surface using the slider mechanism while maintaining light even pressure on both fingers. Subjects were instructed to maintain their finger pressure such that corresponding force indicators on a computer monitor registered values between the numbers 5 and 10 . The values 5 and 10 corresponded to 0.5 and $1.0 \mathrm{~N}$ respectively.

\subsubsection{Screening exercises}

All subjects went through a screening exercise to ensure their ability to perceive out of plane bumps when using the device. Subjects felt several examples of surfaces containing one or two bumps each. Subjects were asked to describe the surfaces in terms of number of bumps as well as their heights. All subjects were able to distinguish bump heights that differed by at least 2 $\mathrm{mm}$ and detect multiple bumps when their spacings were at least $20 \mathrm{~mm}$.

In the case where a single bump is rendered for a single finger, a subject must also be able to determine which finger feels the bump.

Occasionally the apparatus' software controller had to be retuned to a particular subject's hand to ensure stability, but for one subject, the controller could not be adjusted sufficiently. Because sufficient rendering quality could not be achieved, that subject had to be excluded.

\subsubsection{Inter-finger bump calibration}

We have observed in pilot studies that bumps presented to the thumb are often perceived to be smaller than those for the index 
finger. We do not currently have a formal explanation for this phenomenon but are able to calibrate the amplitudes of the two bumps to make them perceptually similar. Using the method of adjustment, subjects manually adjusted the amplitude of a bump displayed only to the thumb so that it matched the perceived amplitude of a bump displayed only to the index finger. The measurement was conducted twice to account for asymmetries from approaching the threshold from the top vs. bottom. Using the touch screen, subjects adjusted the thumb bump's amplitude in steps of $0.075 \mathrm{~mm}$ from a 4 or $8 \mathrm{~mm}$ initial amplitude respectively, until they felt the bumps were equal in size. The average of the two final thumb bump amplitudes was used for all bumps displayed to the thumb for all following experiments. The fixed amplitude used for the index finger was also held constant for future stimulations.

\subsubsection{Experiment 1}

Subjects were told to place their right thumb and index fingers on the two sliders using an open pinch posture (Figure 1). They were instructed to space their two fingers roughly $40 \mathrm{~mm}$ apart and were shown what that spacing looked like. They would then maintain that pose while sliding their fingers along the mechanism's full length several times, feeling a bump with the index finger only. The thumb experienced only a flat surface throughout the motion.

In each trial, after exploring the virtual surface, subjects indicated the location of the perceived bump by pointing to a 160 $\mathrm{mm}$ long horizontal scale on the touch screen. They were told that this length represented the entire explorable range using both fingers together. Subjects were told to focus on the virtual surface as they would for a physical surface (as opposed to attending to their fingers) and to answer based on their mental image.

This procedure was repeated for a total of 30 trials and then again for another 30 with the bump rendered to the thumb alone.

\subsubsection{Experiment 2}

Experiment 2 was similar to Experiment 1 but with the following changes. Subjects were instructed to closely maintain a set finger spacing throughout the experiment. At the start of each trial, a visual cue appeared on the touch screen next to the slider apparatus. The cue consisted of a horizontal bar which changed in length in proportion to the slider separation. The correct slider separation was also indicated on screen. When subjects achieved the correct separation (within $1 \mathrm{~mm}$ of $39.5 \mathrm{~mm}$ ), the visual cue disappeared and the trial began. Subjects then made several passes of the entire length of the workspace while maintaining their finger spacing as closely as possible.

Each slider rendered forces corresponding to one bump along its travel length. The spatial location of the 2 bumps felt separately by each finger could be either the same or $6,12,18,24$, 30,36 or $42 \mathrm{~mm}$ apart. Because the open-pinch posture places the index finger rightward of the thumb, the index finger's bump was always rendered to the right of that of the thumb. Each of the 8 separations was presented 20 times for a total of 160 trials, broken into 4 blocks of 40 trials separated by short break periods. The bump pair's absolute position (measured from the thumb bump) was varied randomly within a $20 \mathrm{~mm}$ range between trials.

In each trial, after exploring the virtual surface, subjects reported the number of bumps, their locations and how many times each bump was encountered. Given that finger spacing is fairly constant, the bump forces could be rendered in one of three ways (Figure 2). Subjects were specifically told that there might be more than one bump per trial and that some bumps might be felt by one finger only. Subjects indicated the location of the perceived bump or bumps by pointing to the same $160 \mathrm{~mm}$ long horizontal scale on the touch screen as before.

After pointing to each bump, two buttons appeared for indicating either 1 or 2 encounters with that particular bump. The instructions for this step were that if a subject ran into a particular bump only once, she was to report one encounter. It was explained that all trials of Experiment 1 were of this type. If she ran into the same bump two separate times, as would occur with a single physical bump, she should report two encounters. To ensure subjects understood the directions, a minimum of 10 practice trials were run where subjects' verbal descriptions of their experiences were compared to their responses.

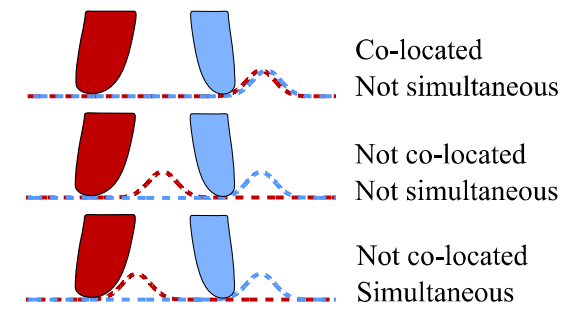

Figure 2. Three potential categories of virtual environments. Each dotted contour represents the surface displayed to the like-colored finger. The left and right fingers as shown represent the thumb and index finger respectively. Some plausible physical analogies are as follows. Top: A single bump which can be encountered twice (once by each finger). Middle: two bumps that can each be encountered once by one finger each. Bottom: same as the previous except bumps are encountered by their respective fingers at the same instant.

\section{Results}

\subsection{Experiment 1}

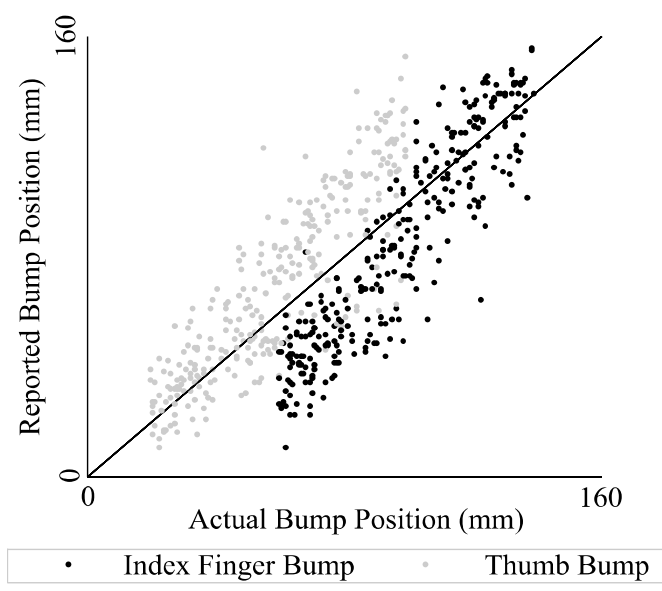

Figure 3. Reported vs. actual bump locations for all subjects in Experiment 1 . Objectively correct response shown by diagonal line.

As shown in Figure 3, subjects exhibited a strong bias to mislocate bumps felt by the thumb and index finger to the right and left, respectively. This bias also increased linearly as fingers approached the middle of the workspace. However the trend continues even beyond the center, suggesting that it is not simply a toward-center bias. The biases were large, resulting in perceived finger locations overlapping or even reversing in order for a given finger spacing. Subtracting this systematic bias by fitting a line to each finger's point cloud and measuring error in the form of residuals, the average standard deviation of errors for 
single-finger bump locating with a pinch posture is $11.3 \mathrm{~mm}$.

\subsection{Experiment 2}

A surprising result was that in trials where two separate bump locations were rendered (separation $>=6 \mathrm{~mm}$ ), subjects responded by envisioning a single bump in $78 \%$ of trials. Clearly, this level of confusion is unlikely on the basis of localization precision, as measured in Experiment 1.
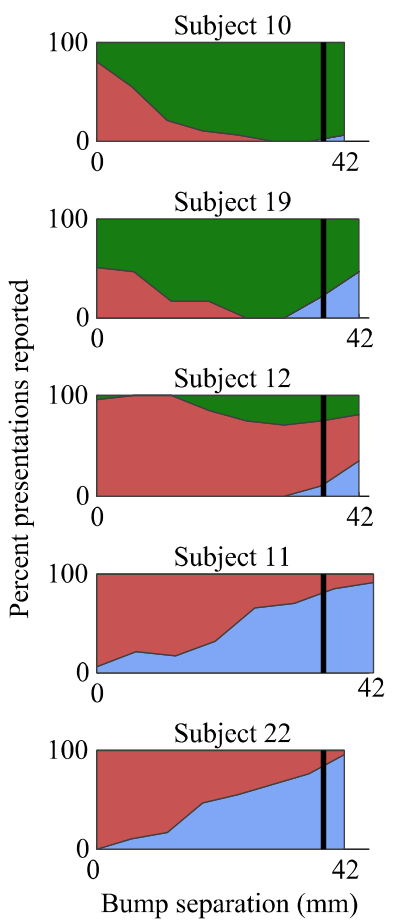

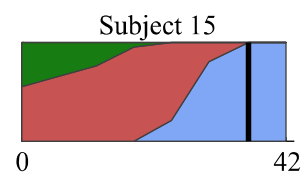

Subject 17
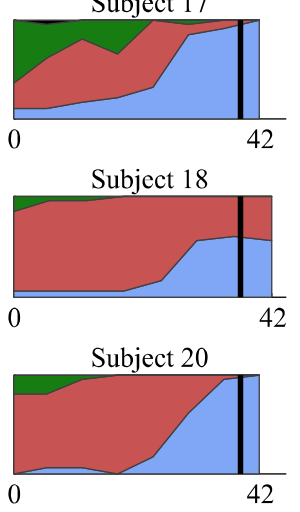

Subject 21

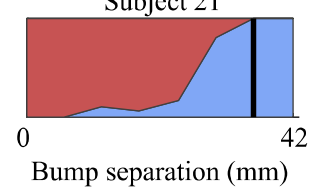

\begin{tabular}{|l|}
1 bump, encountered once \\
1 bump, encountered twice \\
2 bumps, encountered once each \\
\hline
\end{tabular}

Figure 4. Area plots show percentage of trials in Experiment 2, for each bump separation, that correspond to the 3 main virtual surface interpretations. As there were inter-subject variations in the finger positions that affected bump timing, the $x$-axis is scaled so that the spacing corresponding to simultaneous bump encounters for all subjects (shown as a vertical black line) are aligned. Bump separations corresponding to simultaneous bump encounters were calculated using actual finger separation and time data.

Figure 4 shows data from each subject, separated by the class of response (in terms of total number of bumps reported and number of encounters for each). The physical meaning of these response classes are as follows. If the two bumps are rendered such that they occur in the same spatial location (i.e. zero bump separation), then the two fingers encounter the bumps sequentially. This is physically analogous to running into the same physical bump twice, once with each finger. In the context of this experiment, this interaction would be described as a single bump encountered twice. All subjects are able to draw this conclusion at the zero separation with some level of certainty (shown in red). This interpretation declines as the bump separation increases.

If the separation between bumps is non-zero, the analogous physical environment would be two bumps in separate locations encountered once each (by their respective fingers). Each bump effectively disappears before being explored by the other finger. Subjects draw this conclusion at least some of the time (shown in green). Subjects who do make use of this interpretation tend to do so with bump separations at or near zero, although two subjects use it as the predominant response.

If the separation between bumps is the same as the separation between the fingers, the two fingers experience their respective bumps at the same time, shown as the black line in Figure 4. Following the reasoning for the previous case, subjects would respond 2 bumps encountered once each. The only difference is that the bumps are now encountered simultaneously. However this seldom occurs. Instead subjects tend to report a single bump encountered once (shown in blue), a percept that would otherwise only occur if there is only one bump rendered to a single finger.

In fact, 7 of 10 subjects showed a complete dominance of the 1 bump 1 encounter interpretation over the 2 bump 1 encounter per finger (blue vs. green) interpretation when bumps are experienced nearly simultaneously. What this means is that while the fingers are physically stimulated in separate locations, subjects visualize only a single bump on the surface. We refer to this type of response as the collapse of two spatially separate events into a single event. At near-simultaneity of stimulation, only two subjects (10 and 19) consistently envisioned their fingers running over separate bumps simultaneously.

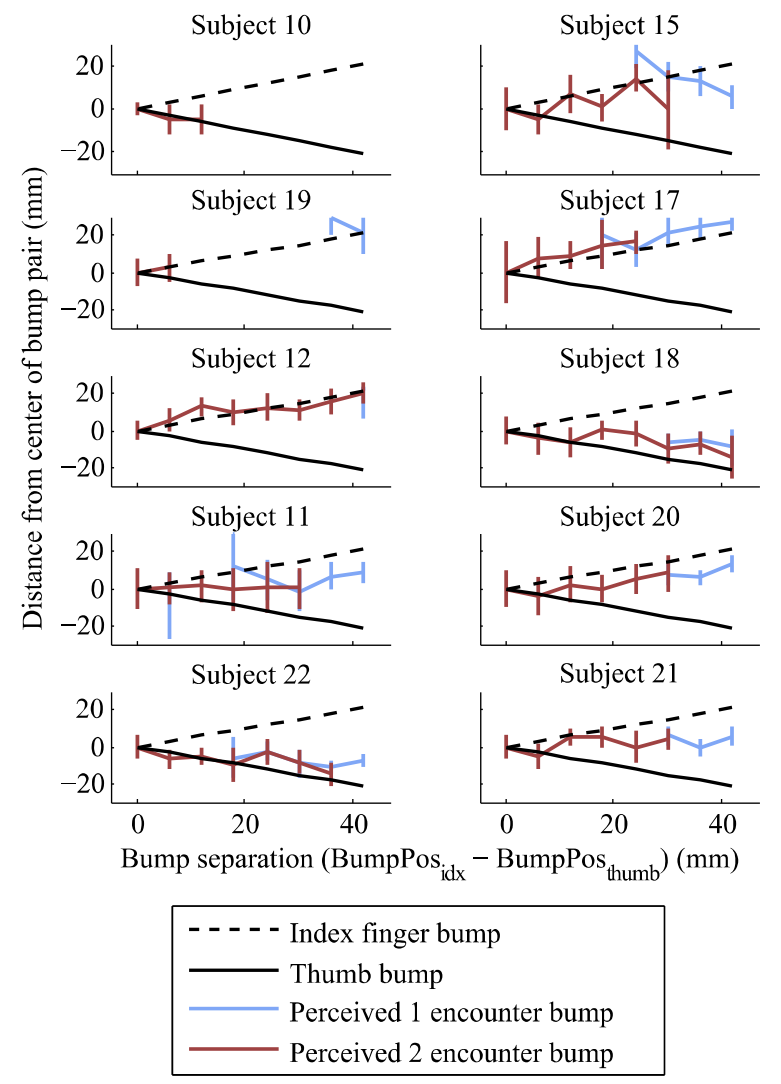

Figure 5. Reported locations of bumps from trials where subjects reported only a single bump. The $y$-axis shows position relative to the midpoint of each rendered bump pair. Error bars denote $95 \%$ confidence intervals and data points computed from fewer than 4 trials are not shown. To account for systematic bias, the data are vertically shifted such that the reported bump location at zero separation coincides with actual bump position.

Figure 5 indicates a mixture of localization trends, with some subjects consistently reporting bump locations aligned with a single finger (primarily index), and the remainder tending to localize bumps between the fingers. Importantly, regardless of the response being biased toward one finger or between them, the trend line established at small spacing, where subjects 
predominantly reported two encounters (consistent with an externalized physical bump that endures across encounters), continues through the transition to larger spacings, where the subjects increasingly reported one encounter (collapse).

\section{Discussion}

\subsection{Experiment 1 bump locating}

In single finger bump locating experiments, subjects show a perceptual bias for locating a given finger closer to the opposite finger. This effect is symmetric for the two fingers and exhibited by most subjects. It is likely that this trend is specific to the situation where two separate fingers travel over a surface, but only one encounters a bump. If the same trend were extended to Experiment 2, it would predict that subjects perceive that their fingers were actually co-located or in some cases reversed in order. This was not observed. What is most relevant to Experiment 2 is that, when this trend is accounted for, residual error in bump localization can be estimated, providing a baseline for error in the second experiment.

Overall bias to shift toward the opposing finger would tend to predict a tendency to report 2 bumps when the stimulations are co-located in space because bias introduces a perceived separation. This does appear at least to a small degree to occur for some subjects in Figure 4 (shown in green).

Experiment 1 also shows that bump localization noise is reasonably low (11.3 $\mathrm{mm}$ standard deviation of error) relative to finger spacing $(39.5 \mathrm{~mm})$. If the spatial cues were unreliable, temporal cues would likely dominate judgments of 1 vs. 2 bumps. Given that the fingers are separated, simultaneity could then present a clue that there are actually two bumps. But as the temporal asynchrony grows, all surfaces might feel like one bump encountered twice (essentially assuming no position information). Our low noise measurement implies that determination of 1 objective bump vs. 2 is based on a combination of both spatial and temporal cues as opposed to temporal only.

\subsection{Experiment 2 bump locating}

Overall, the data in Figure 4 provide strong evidence that when bumps can be felt by only one finger each, one of two interpretations can arise as the spacing of those bumps changes. When the bumps were co-located (zero spacing), subjects almost always interpreted them as separate encounters with the same objective bump. However as the spacing of the bumps approached the spacing between fingers and subjects felt both bumps nearly simultaneously, subjects chose between two interpretations. Two subjects reported feeling separate bumps felt at the same time by both fingers, but the majority of subjects reported only encountering a single bump once. We will begin this discussion by first addressing trials with low or zero bump spacings and then transition to simultaneous bumps and perceptual collapse.

In trials where subjects interpreted their sensations as encountering the same bump with both fingers consecutively, It is likely that the two noisy position estimates were combined in a maximum likelihood fashion (MLE) [13] based on reliability of each location estimate. Simply put, the MLE model states that an estimate of a particular physical parameter is computed as the weighted average of individual estimates derived from each available sensory input. Those weights given to the contributing estimates would be based on their respective reliabilities.

This model would appropriately predict bump locating behavior when the bumps are close enough together to be perceived as the same bump, because MLE models are used to combine estimates of the same objective physical parameter. If reported location estimates then track one finger almost exclusively, this would mean that the total measurement noise in the single-finger location estimate for that finger was much less than that of the other finger. However when the bump separation far exceeds localization noise, subjects are clearly not making repeated estimates of the same physical quantity and the MLE model as described here will no longer be appropriate.

The other alternative would be that the fingers at the same instant are perceived to be coincident, as the data from Experiment 1 appear to suggest at first glance. However as explained earlier, we have reason to doubt that the measured single-finger biases translate directly to two-finger estimates.

The locating biases visible in Figure 5 suggest a common psychophysical computation for locating bumps in both sequentially encountered and simultaneously encountered scenarios. Between surface interpretations, locating biases appear to continue smoothly and linearly with respect to bump spacing. However even if the phenomenological interpretations of simultaneous or non-simultaneous bump encounters may utilize the same low level mechanism for locating the resulting percept, that does not require the two underlying processes themselves (such as grouping) to be the same. An analogous visual example would be locating the centroid of a solid rectangle vs. a set of lines forming a rectangular group (Figure 6). The algorithm for computing the centroid may be the same while the construction of the percepts themselves may rely on separate principles.
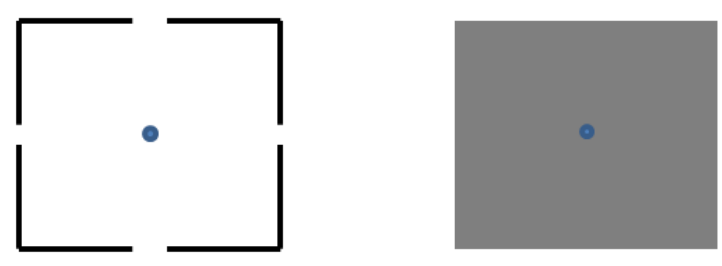

Figure 6. Example of two rectangular shapes that may have common centroid computations but different principles for symbolic construction.

The continuity of localizing trends from the 2 encounter case to the 1 encounter case could alternatively be caused by a bias induced by the experimental protocol. Because the two fingers move collinearly and each pass over just one bump, it is reasonable for a subject to decide a priori that there is in fact only one objective bump (despite being instructed that some bumps might be felt by one finger only). In trials with intermediate spacing, such an a priori conclusion is significantly at odds with the location information received from each encounter. One way of resolving this tension would be to attribute much greater reliability to one finger or the other. This type of bias could then easily manifest in simultaneous bump trials. In the future work section, we will describe an experiment to test this hypothesis.

\subsection{Collapse}

We define perceptual collapse as the dominance of a one-event interpretation (in this case a bump) vs. a multiple-event interpretation (simultaneous but distant bumps) given stimuli in non-contiguous locations. In this experiment, simultaneity and physical spacing are coupled, so it is not yet clear if the effect occurs independently of one or the other factor. It is also not known exactly what causes this type of collapse. Possibilities include grouping phenomena based either on the simplicity of perceptual representations such as the Gestalt principles [5] or on Bayesian priors gathered from experience in the world. In this section we will also consider perceptual masking, which could explain reports of one encounter by suppression of sensations 
originating at one of the two fingers.

\subsubsection{Perceptual grouping}

At the essence of Gestalt philosophy is the law of pragnanz, which states that perception is organized or reduced into the simplest form possible [5]. In other words, if there exists a simple, orderly description that explains the given stimuli as a whole, it should take precedence over a more complex structure that individually describes each perceptual element. In terms of the current experiment, a single bump is certainly structurally simpler than two simultaneously encountered bumps. And because the two bumps are similar in both shape and time, collapsing them into a single bump would sacrifice little descriptive information.

This could very well be an example of haptic common fate, where in this case, multiple force inputs that change in unison, even without a connecting rigid object, are grouped together to describe a single causal event.

Relating this experience to a Bayesian framework, temporally synchronized redundant force information on multiple fingers happens every day in the three-dimensional world, any time we grasp a rigid object. In fact, with the exception of fluid flows, multiple cues from a single rigid object may be one of the only examples of such synchronized force sensations in everyday life. But when we experience these simultaneous forces, we do not attribute causality of these multiple inputs to separate synchronized sources. The same processes may be at work in this two-dimensional scenario. In Bayesian terminology, we likely have strong priors for simultaneous, spatially distinct force stimuli referencing a single source. While not typically discussed in the context of grouping, it is possible that tactile funnelling, which also involves spatial referral to a static location, may belong to the same class of phenomena. In fact, when Lee et al. [12] demonstrated funnelling at a location outside the body, the effect was achieved using a perceived (visual only) instrument that spanned the stimulated fingertips.

\subsubsection{Masking}

It has been shown that for vibrotactile stimuli, one stimulus can be effectively masked by a similar background stimulus in a different location $[14,15]$. This is relevant here because it implies that while stimulations may be simultaneously delivered to multiple fingers, the user may not be able to detect one of those stimulations at all. Consequently, masking presents an alternative interpretation of the observed perceptual collapse because the report of 1 bump encountered once is consistent with subjects only feeling bump forces on a single finger.

However Kim et al. [15] reports that the amplitude ratio of the masking stimulus to the masked stimulus must be on the order of 4 to 5 for two adjacent fingertips. Verillio [14] reported much larger ratios for masking between the thenar eminence and index finger. In the current study, the two stimuli have been calibrated to be almost perceptually equivalent, which casts doubt on the masking hypothesis.

Anecdotally, during practice trials, several subjects spontaneously reported feeling forces on both fingers at once when they felt one bump. For masking to explain their one-bump reports, they would need to feel force sensations on only one finger at a time.

\section{FUTURE WORK}

We are planning an experiment in which bumps are always felt simultaneously but the ratio of the bump amplitudes rendered at the two fingers can vary. This experiment will 1) address the concern mentioned above that subjects locate collapsed bumps nearer to one finger because of bias induced by experience with non-simultaneous, spatially distinct bumps and 2) support or refute masking by determining whether the perceived bump location can be shifted continuously between fingers by varying the relative strengths of the two bump percepts. We also intend to repeat the experiments described here with the addition of a virtual stiffness between the thumb and index finger. We expect that the addition of a virtual object, so to speak, may qualitatively change the perceived interaction into one where the force stimuli are attributed to a single bump explored by the distal end of a virtual tool.

\section{ACKNOWLEDGEMENTS}

This research was supported by the National Science Foundation Graduate Research Fellowship as well as NSF Grant \# IIS0964100.

\section{REFERENCES}

[1] G. Robles-de-la-Torre, Hayward, V., "Force can overcome object geometry in the perception of shape through active touch," Nature, vol. 412, pp. 445-449, 2001.

[2] X. Dai, Colgate, J. E., Peshkin, M. A., "LateralPaD: A surface-haptic device that produces lateral forces on a bare finger," presented at the IEEE Haptics Symposium 2012, Vancouver, BC, Canada, 2012.

[3] E. C. Chubb, Colgate, J.E., Peshkin, M.A., "ShiverPaD: A Glass Haptic Surface That Produces Shear Force on a Bare Finger.," IEEE Transactions on Haptics, vol. 3, pp. 189-198, 2010.

[4] S. G. Manuel, Klatzky, R. L., Peshkin, M. A., Colgate, J. E., "Surface Haptic Feature Attenuation due to Contact on Opposing Surface," presented at the IEEE Haptics Symposium 2012, Vancouver, BC, Canada, 2012.

[5] M. Wertheimer, "(Translated in) Principles of Perceptual Organization," in Readings in Perception, ed: D. Van Nostrand Compnany, 1923/1958, pp. 71-88.

[6] S. E. Palmer, "Perceptual Grouping: It's Later Than You Think," Current Directions in Psychological Science, vol. 11, pp. 101-106, 2002.

[7] A. Gallace, Spence, C., "To What Extent Do Gestalt Grouping Principles Influence Tactile Perception?," Psychological Bulletin, vol. 137, pp. 538-561, 2011.

[8] D. Chang, Nesbitt, K. V., \& Wilkins, K., "The Gestalt principles of similarity and proximity apply to both the haptic and visual grouping of elements," presented at the Conferences in Research and Practice in Information Technology Ballarat, Australia, 2007.

[9] K. E. Overvliet, Krampe, R., Wagemans, J., "Perceptual Grouping in Haptic Search: The Influence of Proximity, Similarity, and Good Continuation," Journal of Experimental Psychology: Human Perception and Performance, vol. 38, pp. 817-821, 2012.

[10] D. Pawluk, Kitada, R., Abramowicz, A., Hamilton, C., Lederman, S. J., "Figure/Ground Segmentation via a Haptic Glance: Attributing Initial Finger Contacts to Objects or Their Supporting Surfaces," IEEE Transactions on Haptics, vol. 4, pp. 2-13, 2011.

[11] M. Miyazaki, Hirashima, M., Nozaki, D., "The "Cutaneous Rabbit" Hopping out of the Body," The Journal of Neuroscience, vol. 30, pp. $1856-1860,2010$.

[12] J. Lee, Kim, Y., Kim, G. J., "Funneling and Saltation Effects for Tactile Interaction with Virtual Objects," presented at the Proceedings of the SIGCHI Conference on Human Factors in Computing Systems, Austin, TX, 2012.

[13] M. O. Ernst, Banks, M.S. , "Humans integrate visual and haptic information in a statistically optimal fashion," Nature, vol. 415, pp. 429-433, 2002.

[14] R. T. Verrillo, Gescheider, G. A., Calman, B. G., Van Doren, C. L., "Vibrotactile masking: Effects of one and two-site stimulation," perception and Psychophysics, vol. 33, pp. 379-387, 1983.

[15] J. R. Kim, Dai, X., Cao, X., Picciotto, C., Tan, D., Tan, H. Z., "A Masking Study of Key-Click Feedback Signals on a Virtual Keyboard," presented at the EuroHaptics 2012, Tampere, Finland, 2012. 